\title{
Hypertrophic pyloric stenosis: tips and tricks for ultrasound diagnosis
}

\author{
Sílvia Costa Dias • Sophie Swinson • Helena Torrão • \\ Lígia Gonçalves • Svitlana Kurochka • Carlos Pina Vaz • \\ Vasco Mendes
}

Received: 5 October 2011 / Revised: 11 February 2012 / Accepted: 19 March 2012 / Published online: 1 May 2012

(C) European Society of Radiology 2012

\begin{abstract}
We describe a systematic approach to the ultrasound (US) examination of the antropyloric region in children. US is the modality of choice for the diagnosis of hypertrophic pyloric stenosis (HPS). The imaging features of the normal pylorus and the diagnostic findings in HPS are reviewed and illustrated in this pictorial essay. Common difficulties in performing the examination and tips to help overcome them will also be discussed.

Main Messages

- Hypertrophic Pyloric Stenosis is defined by thickening of the muscular layer and failure in relaxation of the pyloric canal.

- The main diagnostic criterion is a measurement of more than $3 \mathrm{~mm}$ in thickness of the muscular layer.

- Abnormal elongation of the canal is characterised as greater than $12 \mathrm{~mm}$ in length.
\end{abstract}

Keywords Hypertrophic pyloric stenosis · Ultrasound

S. Costa Dias • H. Torrão $\cdot$ L. Gonçalves $\cdot$ S. Kurochka •

C. P. Vaz $\cdot$ V. Mendes

Radiology Department, Hospital de Braga,

Braga, Portugal

\section{S. Costa Dias $(\square)$}

Rua Pedro Hispano, n 190, 2 Dto,

4100-393 Porto, Portugal

e-mail: silviacostadias@hotmail.com

S. Swinson

Radiology Department, Great Ormond Street

Hospital for Children NHS Trust,

Great Ormond Street,

London WC1N 3JH, UK

\section{Introduction}

Hypertrophic pyloric stenosis (HPS) is the most frequent surgical condition in infants in the first few months of life [1]. The condition is characterised by thickening of the muscular layer and failure of the pyloric canal to relax resulting in gastric outlet obstruction. Elongation of the canal and thickened mucosa are also seen. Ultrasound (US) is the preferred diagnostic modality [2] as it is a non-invasive technique, allowing direct observation of the pyloric canal morphology and behaviour. It is important to carry out a systematic and dynamic study and to be aware of the common technical difficulties and how to overcome them.

\section{Clinical features}

HPS is the most common surgical cause of vomiting in infants. It has an incidence of 3 per 1,000 live births per year, although wide variations have been documented with geographic location, season and ethnic origin [3]. This disease usually presents between the second and sixth weeks of life, more commonly in the white population, in males (male:female ratio 4:1) and typically in first-born children [4]. A history of an affected first-degree relative increases the risk more than five-fold [5].

The US examination allows the radiologist to perform a brief clinical history, which can reveal essential clues to the diagnosis. A recent history of projectile and nonbilious vomiting, which may be intermittent or with every feeding is the classical complaint. Typically the infant has a voracious appetite.

When the vomiting persists, other clinical and biochemical findings may occur such as dehydration, hypochloraemic 
alkalosis and unconjugated jaundice. Late clinical manifestations include weight loss and visible gastric peristaltic activity with a palpable pyloric 'olive'. Prompt US diagnosis is important as these late findings make the infants sub-optimal candidates for surgery.

\section{Imaging technique}

US is the first modality of choice when there is clinical suspicion of HPS, as it is non-invasive and does not use radiation, which is a crucial advantage in children. It is also a commonly available with relatively low cost. US also allows a dynamic study with direct observation of the pyloric canal morphology and behaviour. The US should be performed by an experienced radiologist. Having a systematic approach will improve the sensitivity of the technique.

\section{US examination of the antropyloric region}

Before performing the US, some general conditions for examining infants should be addressed, as these can affect the quality of the examination. The key is to keep the baby comfortable, for example with US gel warmed to a suitable ambient temperature. If possible the examination should be performed after a feeding and accompanied by a parent.

A high-frequency transducer adjusted to the size of the patient and the depth of the pylorus should be used. In the majority of the cases a $6-10 \mathrm{MHz}$ linear probe will provide the depth required to visualise the pylorus [5].

\section{Identification of the pylorus}

First step: In the supine position with the transducer in a transverse position and sometimes with slight anti-clockwise

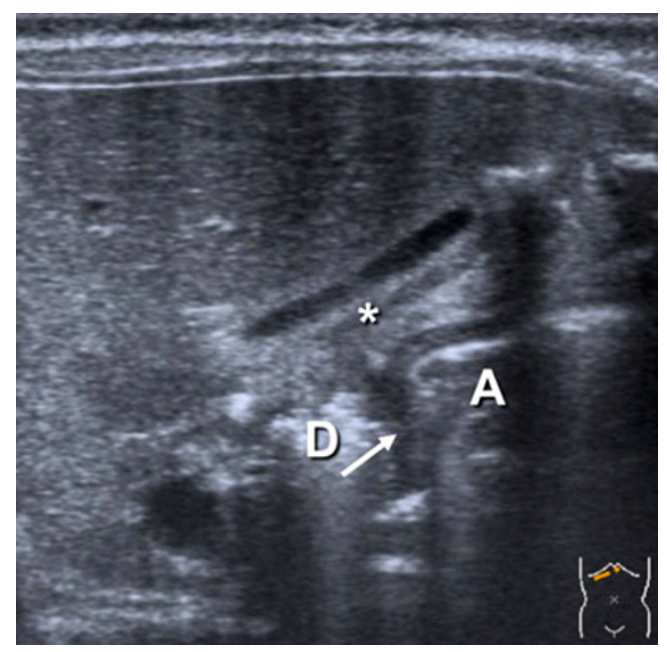

Fig. 1 The pylorus (arrow) between the gastric antrum $(A)$ and the duodenum $(D)$ lying posterior to the gallbladder $\left(^{*}\right)$

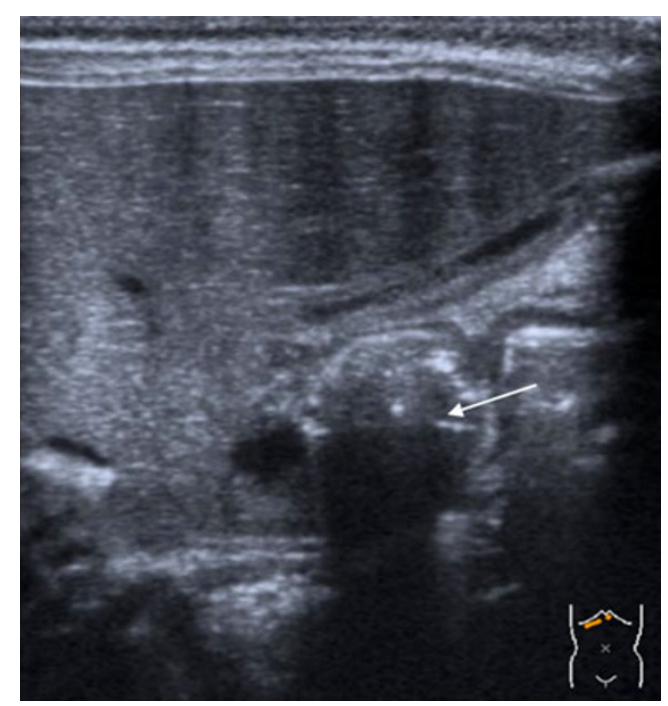

Fig. 2 Passage of the gastric content through the pylorus, distending the antropyloric region (arrow)

rotation, identify the gallbladder. The pylorus is usually located slightly medial and posterior in relation to the gallbladder (Fig. 1).

\section{Observe the pyloric morphology}

Second step: Assess the appearance and measurements of the pylorus (Fig. 1). The muscular layer is usually a hypoechogenic thin layer less than $2 \mathrm{~mm}$ in thickness. It is important to be aware that tangential views and contractions can produce pseudo-thickening.

\section{Observe the pyloric behaviour}

Third step: Visualize the passage of the gastric content through the pylorus, distending the antropyloric region. This dynamic evaluation is vital, as a wide open pylorus with normal passage of the gastric contents excludes HPS (Fig. 2).

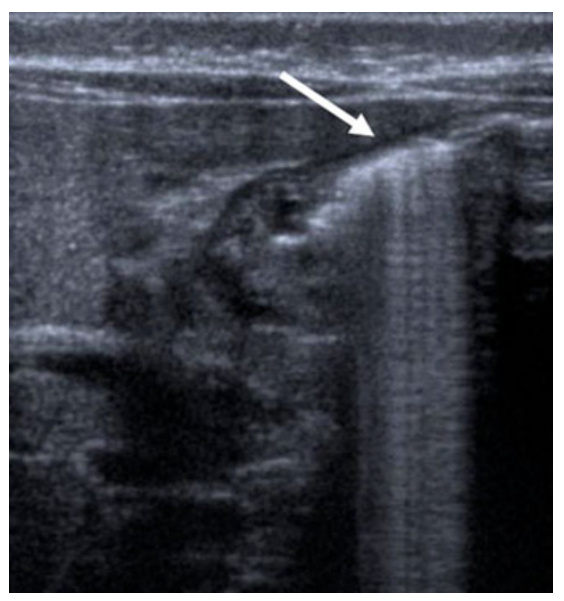

Fig. 3 The stomach distended with gas (arrow) 


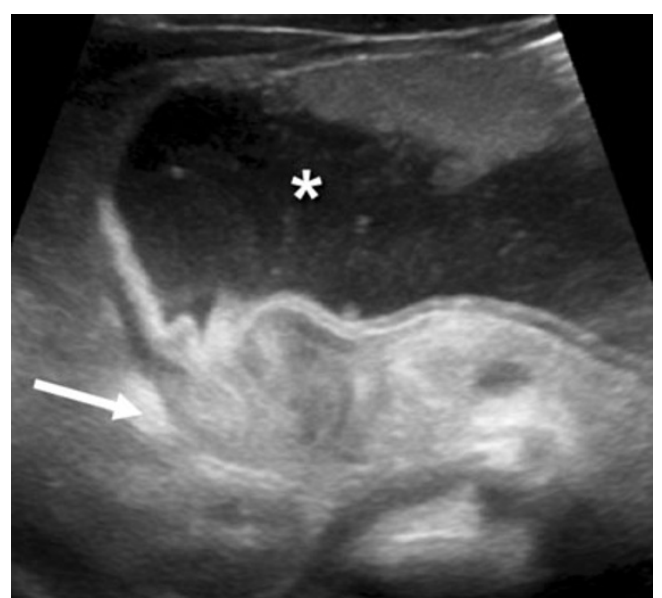

Fig. 4 The distended stomach $(*)$, posteriorly displacing the pylorus (arrow), which resembles the appearance of the uterine cervix

Tips and tricks

One common difficulty is a stomach filled with gas (Fig. 3). The easiest way to avoid this is by placing the infant in an oblique position with the right side down, as this will allow fluid to fill the antrum, acting as an acoustic window. A stomach completely filled with milk can also cause artefacts, other possibilities are to give the infant water or even to place a nasogastric tube, empty the stomach and then fill it with water.

Another frequent problem is that a markedly distended stomach can displace the pylorus dorsally making it very difficult to access (Fig. 4). In this situation, moving the infant into an oblique position with the left side down will help to move the pylorus to a more anterior position.

The identification of the pylorus can be difficult, but a systematic approach will improve chances of success. Remember that a normal pylorus is much harder to visualise than a hypertrophied one.

\section{US diagnostic criteria of HPS}

The main diagnostic criterion is measurement of the thickness of the muscular layer. An abnormal cut off value of

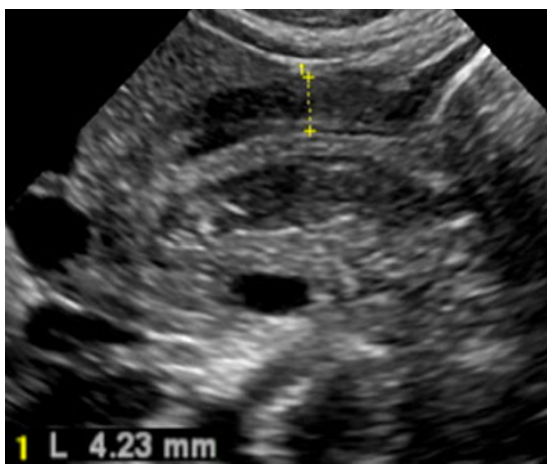

Fig. 5 The hypertrophied muscular layer

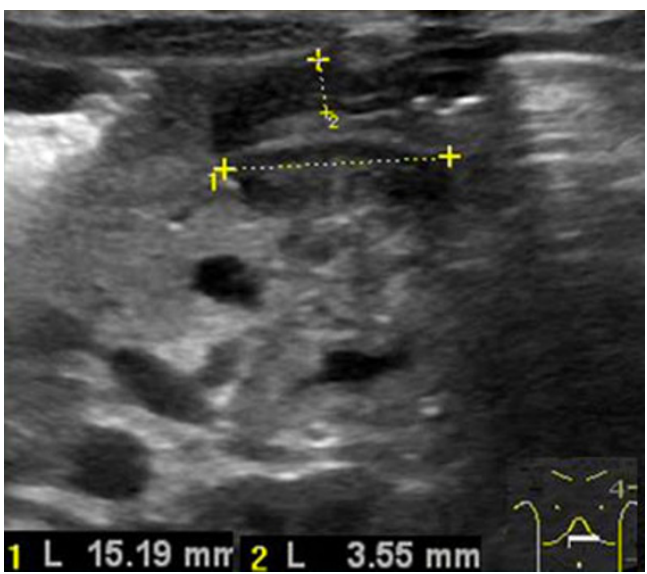

Fig. 6 Abnormal elongation of the pyloric canal (measure 1)

$3 \mathrm{~mm}$ in thickness has been described in the literature (Figs. 5 and 6) [6-9].

The other principal sonographic size criterion is the length of the pyloric canal. Abnormal elongation of the canal is defined as greater than $12 \mathrm{~mm}$ in length (Fig. 6) [9], however this measure is more difficult to perform and for this reason is considered a less reliable criterion [10].

In HPS the thickened muscle and elongated pylorus are fixed over time, which helps the operator to identify this condition. The appearance of the hypertrophied pylorus has previously been described as the cervix sign [11], as it resembles the appearance of the uterine cervix (Fig. 4).
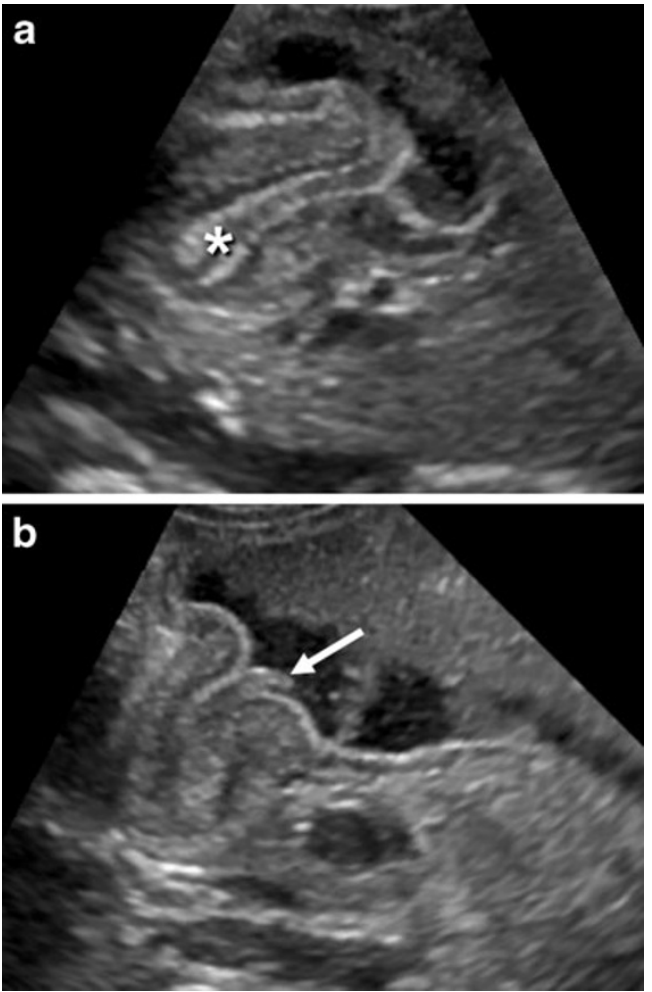

Fig. 7 a The double layer of thickened mucosa $(*)$, b protruding through the antrum 
Additional US findings in HPS are hypertrophy of the mucosa and a markedly distended and actively peristalsing stomach. A double internal layer of crowded and redundant mucosa may be identified (Fig. 7a), protruding through the antrum (Fig. 7b). This was classically described as the nipple sign in conventional contrast studies. The double layer of thickened mucosa is hyperechogenic and can be confused with echogenic contents passing through the pylorus.

\section{Borderline measures}

Thickening of the pyloric canal may be transient due to peristalsis or pylorospasm. In the majority of cases of pylorospasm, the muscle is not hypertrophied. Sometimes the muscle can be slightly thick, but it usually measures less than $3 \mathrm{~mm}$. With prolonged observation, pyloric opening may be visualised.

If the muscle layer is $2-3 \mathrm{~mm}$ thick, and it does not relax during examination, clinical follow-up with repeat US is advisable $[5,6,12]$. Particular attention should be paid to pre-term infants and those in the younger age range. In premature infants, HPS develops at the same age as in term infants, but their smaller size should be taken into consideration. Argyropoulou et al. [13] showed that normal pyloric dimensions increase with the gestational age and documented an even stronger correlation with body weight, providing normal values for muscle thickness, canal length and canal width from prematurity to full-term infants. Haider et al. [14] performed a study with 190 infants operated for HPS and found a strong correlation with the ultrasound measurement of the pyloric length and the weight of the infant, which can be helpful in small and premature patients. However these authors also highlight the importance of the morphological appearance of the pylorus in premature infants.

\section{Post-treatment imaging of HPS}

The treatment of HPS is surgical pyloromyotomy. A further US examination may be requested if vomiting persists following surgery. However, the radiologist and the surgeon should be aware that the pyloric muscle may remain thickened after successful surgery and can take up to 5 months to return to normal thickness.

In the first week after surgery, the muscle can be the same thickness or even thicker than before surgery and then the dimensions gradually return to normal. The anterior part of the muscle tends to normalize first, usually measuring less than $3 \mathrm{~mm}$ by 3 months. The posterior part is last to normalise, usually after 5 months, when the pylorus regains its appearance of an elongated ring. This order of changes is related to the anterior surgical approach to the muscle [15].

An upper GI examination may also be performed if emesis continues post-operatively, in order to exclude a duodenal leak or to assess an incomplete pyloromyotomy or gastro-oesophageal reflux [16].

\section{Conclusion}

Pyloric US examination is a dynamic investigation, which should be performed in a systematic way. The radiologist should be aware of the pitfalls of the examination and how to overcome them. It is important to be familiar with the normal and hypertrophied pyloric appearances, as this will provide a greater diagnostic confidence, assisting in early diagnosis and improving the management of infants with HPS.

\section{References}

1. Ohshiro K, Puri P (1998) Pathogenesis of infantile hypertrophic pyloric stenosis: recent progress. Pediatr Surg Int 13:43-252

2. Hiorns MP (2011) Gastrointestinal tract imaging in children: current techniques. Pediatr Radiol 41:42-54

3. Panteli C (2009) New insights into the pathogenesis of infantile pyloric stenosis. Pediatr Surg Int 25:1043-1052

4. Chandran L, Chitkara M (2008) Vomiting in children: reassurance, red flag, or referral? Pediatr Rev 29:183-192

5. Hernanz-Schulman M (2003) Infantile hypertrophic pyloric stenosis. Radiology 227:319-331

6. O'Keeffe FN, Stansberry SD, Swischuk LE, Hayden CK (1991) Antropiloric muscle thickness at US in infants: what is normal. Radiology 178:827-830

7. Hernanz-Schulman M, Sells LL, Ambrosino MM, Heller RM, Stein SM, Neblett WW (1994) Hypertrophic pyloric stenosis in the infant without a palpable olive: accuracy of sonographic diagnosis. Radiology 193:771-776

8. Rohrschneider WK, Mittnacht H, Darge K, Tröger J (1998) Pyloric muscle in asymptomatic infants: sonographic evaluation and discrimination from idiopathic hypertrophic pyloric stenosis. Pediatr Radiol 28:429-434

9. Reed AA, Michael K (2010) Hypertrophic pyloric stenosis. J Diagn Med Sonography 26:157-160

10. Blumhagen JD, Maclin L, Krauter D, Rosenbaum DM, Weinberger E (1988) Sonographic diagnosis of hypertrophic pyloric stenosis. AJR Am J Roentgenol 150:1367-1370

11. Haller JO, Cohen HL (1986) Hypertrophic pyloric stenosis: diagnosis using US. Radiology 161:335-339

12. Hernanz-Schulman M (2009) Pyloric stenosis: role of imaging. Pediatr Radiol 39(Suppl 2):S134-S139

13. Argyropoulou MI, Hadjigeorgi CG, Kiortsis DN (1998) Antro-pyloric canal values from early prematurity to full-term gestational age: an ultrasound study. Pediatr Radiol 28:933-936

14. Haider N, Spicer R, Grie D (2002) Ultrasound diagnosis of infantile hypertrophic pyloric stenosis: determinants of pyloric length and the effect of prematurity. Clin Radiol 57:136-139

15. Yoshizawa J, Eto T, Higashimoto Y, Saitou T, Maie M (2001) Ultrasonographic features of normalization of the pylorus after pyloromyotomy for hypertrophic pyloric stenosis. J Pediatr Surg 36:582-586

16. Nasr A, Ein SH, Connolly B (2008) Recurrent pyloric stenosis: to dilate or operate? A preliminary report. J Pediatr Surg 43:E17-E20 